\title{
SOLUTIONS TO $\psi$-TYPE COMPLEX ORDER DELAY DIFFERENTIAL EQUATIONS WITH NONLOCAL INITIAL CONDITIONS
}

\author{
D. VIVEK ${ }^{1}$, E. M. ELSAYED ${ }^{2, *}$, K. KANAGARAJAN ${ }^{3}$, J. PRASANTH ${ }^{1}$ \\ ${ }^{1}$ Department of Mathematics, PSG College of Arts \& Science, Coimbatore-641014, India \\ ${ }^{2}$ Department of Mathematics, Faculty of Science,King Abdulaziz University, Jeddah 21589, Saudi Arabia \\ ${ }^{3}$ Department of Mathematics, Sri Ramakrishna Mission Vidyalaya College of Arts and Science, \\ Coimbatore-641020, India \\ Corresponding author: emmelsayed@yahoo.com
}

Received Apr. 14, 2020

\begin{abstract}
Авstract. In this paper, we focus on the existence and uniqueness of solutions for a class of $\psi$-type complex-order delay differential equations subject to nonlocal initial conditions. By employing Krasnoselkii's fixed point theorem, the conditions for the existence of solutions are established in Banach space.
\end{abstract}

2010 Mathematics Subject Classification. 34A12; 26E50; 45G10.

Key words and phrases. $\psi$-fractional derivative; nonlocal conditions; delay differential equations; existence; fixed point.

\section{InTRODUCTION}

Throughout this paper $(X,\|\cdot\|)$ will be a Banach space, and $J:=[0, T], T>0$, a compact interval in $\mathbb{R}$. Denote by $C(J, X)$ the Banach space of all continuous functions $J \rightarrow X$ endowed with the topology of uniform convergence (the norm in this space will be denoted by $\|\cdot\|_{C}$ ).

The purpose of this paper is to establish some new results on existence and uniqueness of solutions for the following $\psi$-type complex order delay differential equations (DDEs) with nonlocal initial condition

$$
\left\{\begin{array}{l}
\left(\mathscr{D}^{\theta ; \psi} x\right)(t)=\mathscr{F}\left(t, x\left(\sigma_{1}(t)\right), x\left(\sigma_{2}(t)\right), \ldots, x\left(\sigma_{n}(t)\right)\right), \quad t \in J, \\
x(t)+g\left(t_{1}, t_{2}, \ldots, t_{p}, x(\cdot)\right)=x_{0},
\end{array}\right.
$$

DOI: 10.28924/APJM/7-14 
where $\mathscr{D}^{\theta ; \psi} x$ is the $\psi$-type Caputo fractional order of order $\theta \in \mathbb{C}, \theta=m+i \alpha$. Let $\alpha \in \mathbb{R}^{+}$, $0<\alpha<1, m \in(0,1]$ and $\mathscr{F}: J \times \mathbb{R}^{n} \rightarrow \mathbb{R}, g: J^{p} \times \mathbb{R} \rightarrow \mathbb{R}, \sigma_{i}=J \times J(i=1, \ldots, n)$ are given continuous functions.

It is observed that equation (1.1) is equivalent to the integral equation

$$
x(t)=\left\{\begin{array}{l}
x_{0}-g\left(t_{1}, t_{2}, \ldots, t_{p}, x(\cdot)\right) \\
+\frac{1}{\Gamma(\theta)} \int_{0}^{t} \psi^{\prime}(s)(\psi(t)-\psi(s))^{\theta-1} \\
\times \mathscr{F}\left(s, x\left(\sigma_{1}(s)\right), x\left(\sigma_{2}(s)\right), \ldots, x\left(\sigma_{n}(s)\right)\right) d s .
\end{array}\right.
$$

Recent study of complex order differential equations are done by Vivek et al. in the papers [7-10]. Almeida introduced the $\psi$-type Caputo fractional derivative of a function in [2]. In passing, we remark that the application of nonlinear condition $x(0)=x_{0}-g\left(t_{1}, t_{2}, \ldots, t_{p}, x(\cdot)\right)$ in physical problems yields better effect than the initial condition $x(0)=x_{0}$, for detailed informations $[1,5]$.

On the other hand, this type of DDEs have also been studied by Balachandran, see $[3,4]$. But, as far as we know, papers considering the fractional-order DDEs are seldom. The main contributions of this work are as follows:

(i) We introduce $\psi$-type complex order derivative.

(ii) We establish a sufficient condition for the existence of solutions to the proposed problem (1.1).

The rest of this paper is organized as follows. In Section 2, some preliminaries are given on the $\psi$-complex order derivatives. In Section 3, we study the existence and uniqueness of solutions of the problem (1.1).

\section{Preliminaries}

In this section, we introduce some basic facts as regards the $\psi$-type complex order derivative and fractional calculus.

Definition 2.1. The $\psi$-type Riemann-Liouville fractional integral of order $\theta \in \mathbb{C},(\operatorname{Re}(\theta)>0)$ of a function $\mathscr{F}:(0, \infty) \rightarrow \mathbb{R}$ is

$$
\mathscr{I}^{\theta ; \psi} \mathscr{F}(t)=\frac{1}{\Gamma(\theta)} \int_{0}^{t} \psi^{\prime}(s)(\psi(t)-\psi(s))^{\theta-1} \mathscr{F}(s) d s
$$


Definition 2.2. For a function $\mathscr{F}$ given by on the interval $J$, the $\psi$-type Caputo fractional-order $\theta \in \mathbb{C}$, $(\operatorname{Re}(\theta)>0)$ of $\mathbb{R}$, is defined by

$$
\left(\mathscr{D}^{\theta ; \psi} \mathscr{F}\right)(t)=\frac{1}{\Gamma(n-\theta)} \int_{0}^{t} \psi^{\prime}(s)(\psi(t)-\psi(s))^{n-\theta-1} \mathscr{F}(s) d s,
$$

when $n=[\operatorname{Re}(\theta)]+1$ and $[\operatorname{Re}(\theta)]$ denotes the integral part of the real number $\nu$.

Definition 2.3. [6] The Stirling asymptotic formula of the Gamma function for $z \in \mathbb{C}$ is following

$$
\Gamma(z)=(2 \pi)^{\frac{1}{2}} z^{\frac{z-1}{2}} e^{-z}\left[1+O\left(\frac{1}{z}\right)\right], \quad(|\arg (z)|<\pi ;|z| \rightarrow \infty),
$$

and its results for $|\Gamma(u+i v)|,(u, v \in \mathbb{R})$ is

$$
|\Gamma(u+i v)|=(2 \pi)^{\frac{1}{2}}|v|^{u-\frac{1}{2}} e^{-u-\pi|v| / 2}\left[1+O\left(\frac{1}{v}\right)\right], \quad(v \rightarrow \infty) .
$$

Now, we state a known result due to Krasnoselskii which is needed to prove the existence of at least one solution of (1.1).

Theorem 2.1. Let $M$ be closed convex and nonempty subset of a Banch space $X$. Let $A, B$ be two operators such that

(i) $A x+B y \in M$ whenever $x, y \in M$;

(ii) $A$ is compact and continuous;

(iii) $B$ is a contraction mapping.

Then there exists $z_{1} \in M$ such that $z_{1}=A z_{1}+B z_{1}$.

\section{EXISTENCE OF SOLUTIONS}

In this section, we introduce the existence theorems of solutions of the problem (1.1).The discussions are based on fixed point theorems. Our main results are as follows.

For convenience, we list the following hypotheses:

(H1) $\mathscr{F}: J \times \mathbb{R}^{n} \rightarrow \mathbb{R}$ is jointly continuous.

(H2) There exists a Lipschitz constant $L^{*}>0$ such that

$$
\begin{aligned}
& \left|\mathscr{F}\left(t, x_{1}, x_{2}, \ldots, x_{n}\right)-\mathscr{F}\left(t, y_{1}, y_{2}, \ldots, y_{n}\right)\right| \\
& \leq L^{*}\left\{\left|x_{1}-y_{1}\right|+\left|x_{2}-y_{2}\right|+\ldots+\left|x_{n}-y_{n}\right|\right\},
\end{aligned}
$$

for $t \in J, x_{i}, y_{i} \in X,(i=1,2, \ldots, n)$;

(H3) $g: J^{p} \times \mathbb{R} \rightarrow \mathbb{R}$ is continuous and there exists a constant $b>0$ such that

$$
\left|g\left(t_{1}, t_{2}, \ldots, t_{p}, x(\cdot)\right)-g\left(t_{1}, t_{2}, \ldots, t_{p}, y(\cdot)\right)\right| \leq b|x-y|, \quad \forall x, y \in X .
$$


(H4) $\left|\mathscr{F}\left(t, x_{1}, x_{2}, \ldots, x_{n}\right)\right| \leq \mu(t)$, for all $\left(t, x_{i}\right) \in J \times X,(i=1,2, . ., n)$, where $\mu \in L^{1}\left(J, \mathbb{R}^{+}\right)$.

Theorem 3.1. Assume that hypotheses (H1)-(H3) are fulfilled. If $\Omega_{b, L^{*}, \theta}<1$, then the problem (1.1) has a unique solution on $J$.

Proof. Define $C(J, X) \rightarrow C(J, X)$ by

$$
(N x)(t)=\left\{\begin{array}{l}
x_{0}-g\left(t_{1}, t_{2}, \ldots, t_{p}, x(\cdot)\right) \\
+\frac{1}{\Gamma(\theta)} \int_{0}^{t} \psi^{\prime}(s)(\psi(t)-\psi(s))^{\theta-1} \\
\times \mathscr{F}\left(s, x\left(\sigma_{1}(s)\right), x\left(\sigma_{2}(s)\right), \ldots, x\left(\sigma_{n}(s)\right)\right) d s .
\end{array}\right.
$$

Choose $r \geq 2\left(\left\|x_{0}\right\|+G+\left(n L^{*} r+M^{*}\right) \frac{(\psi(T))^{m}}{m|\Gamma(\theta)|}\right)$, and let $\sup _{t \in J}|f(t, 0, \ldots, 0)|=M^{*}$. Then we can show that $N B_{r} \subset B_{r}$, where $B_{r}:=\{x \in C(J, X):\|x\| \leq r\}$.

So let $x \in B_{r}$ and set $G=\sup _{x \in C(J, X)}\left|g\left(t_{1}, t_{2}, \ldots, t_{p}, x(\cdot)\right)\right|$. Then we get

$$
\begin{aligned}
& |(N x)(t)| \\
& \leq\left|x_{0}\right|+G+\frac{1}{\Gamma(\theta)} \int_{0}^{t} \mid \psi^{\prime}(s)(\psi(t)-\psi(s))^{\theta-1} \\
& \quad \times \mathscr{F}\left(s, x\left(\sigma_{1}(s)\right), x\left(\sigma_{2}(s)\right), \ldots, x\left(\sigma_{n}(s)\right)\right) \mid d s \\
& \leq\left|x_{0}\right|+G+\frac{1}{\Gamma(\theta)} \int_{0}^{t}\left|\psi^{\prime}(s)(\psi(t)-\psi(s))^{\theta-1}\right| \\
& \times\left(\mid \mathscr{F}\left(s, x\left(\sigma_{1}(s)\right), x\left(\sigma_{2}(s)\right), \ldots, x\left(\sigma_{n}(s)\right)\right)\right. \\
& \quad-f(t, 0,0, \ldots, 0)|+| f(t, 0,0, \ldots, 0) \mid) d s \\
& \leq\left|x_{0}\right|+G+\left(n L^{*} r+M^{*}\right) \frac{1}{|\Gamma(\theta)|} \int_{0}^{t}\left|\psi(s)(\psi(t)-\psi(s))^{\theta-1}\right| d s \\
& \leq\left|x_{0}\right|+G+\left(n L^{*} r+M^{*}\right) \frac{(\psi(T))^{m}}{m|\Gamma(\theta)|} \\
& \leq r
\end{aligned}
$$

by choice of $L^{*}$ and $r$. Now take $x, y \in C(J, X)$. Then we get

$$
\begin{aligned}
& |(N x)(t)-(N y)(t)| \\
& \leq|g(x)-g(y)|+\frac{1}{|\Gamma(\theta)|} \int_{0}^{t}\left|\psi^{\prime}(s)(\psi(t)-\psi(s))^{\theta-1}\right| \mid \mathscr{F}\left(s, x\left(\sigma_{1}(s)\right), x\left(\sigma_{2}(s)\right), \ldots, x\left(\sigma_{n}(s)\right)\right) \\
& -\mathscr{F}\left(s, y\left(\sigma_{1}(s)\right), y\left(\sigma_{2}(s)\right), \ldots, y\left(\sigma_{n}(s)\right)\right) \mid d s \\
& \leq\left(b+\frac{n L^{*}(\psi(T))^{m}}{m|\Gamma(\theta)|}\right)\|x-y\| .
\end{aligned}
$$


Thus

$$
\|N x-N y\| \leq\left(b+\frac{n L^{*}(\psi(T))^{m}}{m|\Gamma(\theta)|}\right)\|x-y\|,
$$

where $\Omega_{b, L^{*}, \theta}=\left(b+\frac{n L^{*}(\psi(T))^{m}}{m|\Gamma(\theta)|}\right)$ depends only on the parameters of the problem. And since $\Omega_{b, L^{*}, \theta}<1$, the result follows in view of the contraction mapping principle.

Theorem 3.2. Assume that (H1), (H3) and (H4) are fulfilled. Then the problem (1.1) has at least one solution on $J$.

Proof. Choose $r \geq\left\|x_{0}\right\|+G+\frac{(\psi(T))^{m}\|\mu\|_{L^{1}}}{m|\Gamma(\theta)|}$. Now define on $B_{r}$ the operators $A, B$ by

$$
\begin{aligned}
(A x)(t):= & \frac{1}{\Gamma(\theta)} \int_{0}^{t} \psi^{\prime}(s)(\psi(t)-\psi(s))^{\theta-1} \\
& \times \mathscr{F}\left(s, x\left(\sigma_{1}(s)\right), x\left(\sigma_{2}(s)\right), \ldots, x\left(\sigma_{n}(s)\right)\right) d s,
\end{aligned}
$$

and

$$
(B x)(t):=x_{0}-g\left(t_{1}, t_{2}, \ldots, t_{p}, x(\cdot)\right) .
$$

Let's observe that if $x, y \in B_{r}$, then $A x+B y \in B_{r}$. Indeed it is easy to check the inequality

$$
\|A x+B y\| \leq\left|x_{0}\right|+G+\frac{(\psi(T))^{m}\|\mu\|_{L^{1}}}{m|\Gamma(\theta)|} \leq r .
$$

By (H3), it is also clear that $B$ is a contraction mapping for $b<1$.

Since $x$ is continuous, then $(A x)(t)$ is continuous in view of $(\mathrm{H} 1)$. Let's now note that $A$ is uniformly bounded on $B_{r}$. This follows from the inequality

$$
\|(A x)(t)\| \leq \frac{(\psi(T))^{m}\|\mu\|_{L^{1}}}{m|\Gamma(\theta)|} .
$$

Now let's prove that $(A x)(t)$ is equicontinuous.

Let $t_{1}, t_{2} \in J$ and $x \in B_{r}$. Using the fact that $\mathscr{F}$ is bounded on the compact set $J \times B_{r}$. (Thus $\left.\sup _{(t, x) \in J \times B_{r}}\left\|\mathscr{F}\left(t, x\left(\sigma_{1}(x)\right), x\left(\sigma_{2}(x)\right), \ldots, x\left(\sigma_{n}(x)\right)\right)\right\|:=c_{0}<\infty\right)$, we get

$$
\begin{aligned}
& \left|(A x)\left(t_{1}\right)-(A x)\left(t_{2}\right)\right| \\
& \leq \frac{1}{|\Gamma(\theta)|} \mid \int_{0}^{t_{1}} \psi^{\prime}(s)\left(\psi\left(t_{1}\right)-\psi(s)\right)^{\theta-1} \mathscr{F}\left(t, x\left(\sigma_{1}(x)\right), x\left(\sigma_{2}(x)\right), \ldots, x\left(\sigma_{n}(x)\right)\right) \\
& -\int_{0}^{t_{2}} \psi^{\prime}(s)\left(\psi\left(t_{2}\right)-\psi(s)\right)^{\theta-1} \mathscr{F}\left(t, x\left(\sigma_{1}(x)\right), x\left(\sigma_{2}(x)\right), \ldots, x\left(\sigma_{n}(x)\right)\right) \mid \\
& \leq \frac{c_{0}}{|\Gamma(\theta)|}\left|2\left(\psi\left(t_{1}\right)-\psi\left(t_{1}\right)\right)^{\theta}+\left(\psi\left(t_{2}\right)\right)^{\theta}-\left(\psi\left(t_{1}\right)\right)^{\theta}\right|
\end{aligned}
$$


which tends to zero as $t_{1}-t_{2} \rightarrow 0$. Hence along with the Arzela-Ascoli theorem $A$ is compact. We now conclude the result of the theorem based on the Krasnoselkii's theorem above.

\section{ACKNOWLEDGEMENT}

The authors are grateful to the referees for their careful reading of the manuscript and valuable comments. The authors thank the help from the editor too.

\section{Authors' CONTRIBUtions}

All authors contributed equally and significantly in writing this article. All authors read and approved the final manuscript.

\section{REFERENCES}

[1] B. Ahmad, S. Sivasundaram, Some existence results for fractional integro-differential equations with nonlocal conditions, Commun. Appl. Anal., 12(2) (2008), 107-112.

[2] R. Almeida, A Caputo fractional derivative of a function with respect to another function, Commun. Nonlinear Sci. Numer. Simul., 44 (2017), 460-481.

[3] K. Balachandran, M. Chandrasekaran, Existence of solutions of a delay differential equation with nonlocal condition, Indian J. Pure Appl. Math., 27(5) (1996), 443-449.

[4] K. Balachandran, P. Prakash, Existence of solutions of fuzzy delay differential equations with nonlocal condition, J. Korean Soc. Industr. Appl. Math., 6(1) (2002), 81-89.

[5] G. M. Guerekata, A Cauchy problem for some fractional abstract differential equation with nonlocal conditions, Nonlinear Anal., 70 (2009), 1873-1876.

[6] A. A. Kilbas, H. M. Srivasta, J. J. Trujillo, Theory and Application of Fractional Differential Equations, Elsevier B. V, Netherlands (2016).

[7] D. Vivek, E.M. Elsayed, K. Kanagarajan, Existence and Ulam stability results for a class of boundary value problem of neutral pantograph equations with complex order, SeMA. (2020). https://doi .org/10.1007/ s40324-020-00214-1.

[8] O. Baghani, D. Vivek, K. Kanagarajan, Theory of hybrid fractional differential equations with complex order, Sahand Commun. Math. Anal., 15(1) (2019), 65-76.

[9] D. Vivek, K. Kanagarajan, S. Harikrishnan, Dynamics and Stability Results of Fractional Pantograph Equations with Complex Order, J. Appl. Nonlinear Dyn. , 7(2) (2018) 179-187.

[10] D. Vivek, K. Kanagarajan, S. Harikrishnan, Dynamics and stability results of fractional integro-differential equations with complex order, Discontin. Nonlinearity Complex., 7(2) (2018), 119-127. 